\title{
Falling from Digital Grace: Participation in Online Software Contests Following Loss of Status
}

\author{
Swanand J Deodhar \\ Indian Institute of Management, \\ Ahmedabad \\ swanandd@iima.ac.in
}

\author{
Yash Babar \\ Carlson School of Management \\ University of Minnesota \\ babar008@umn.edu
}

\author{
Gordon Burtch \\ Carlson School of Management \\ University of Minnesota \\ gburtch@umn.edu
}

\begin{abstract}
We focus on the effects of status loss on decisions to participate in subsequent contests in online coding platform. We advance the relevant literature in several ways. First, by considering the effects of status loss on resource expenditure, we depart from the prior status literature, which has predominantly looked at performance implications of the status loss. Second, because of the voluntary nature of online contests, we demonstrate how the effects of status loss manifest when permanent exit or abstention is possible. This aspect marks another departure from situations common to the prior work, wherein work demands persist regardless of status changes. Lastly, recognizing that status changes may be endogenous to one's past resource expenditure, we study exogenous variation in status, exploiting a natural experiment wherein status assignments were adjusted overnight by the platform operator, in a manner completely independent of individuals' prior activities, resulting in sudden loss of status.
\end{abstract}

\section{Introduction}

Since Merton's seminal paper [1], management scholars have extensively studied the effects of status, which often manifests as an "ordering or ranking in a social system" [2; p. 284]. Prior studies collectively suggest that holding high status is desirable because individuals who possess such status can reap significantly more economic and social benefits than those who do not [3]. However, an individual's status is not permanent. Status may be lost because of a variety of reasons. For example, prior work has explored situations in which status was lost because of a public scandal involving a highstatus individual [4], as well as when a person faces a loss of favorable endorsement from a third-party agency [5]. Regardless of why it occurs, the status loss can significantly alter individuals' behavior [6].
We build on this prior literature by studying the behavioral implications of status loss, resulting from the reordering of the status-defining categories at a platform for online software coding contests. In so doing, our work responds to recent calls for examining how people behave after losing status [7; p. 225].

Extant literature is mostly restricted to explaining the effect of status loss on an individual's or organization's performance in employment or the market. With limited exception involving short duration project teams involving students [8; p. 341], prior work suggests that losing status leads to poorer performance. [7] find that status loss results in inconsistencies between status and the individual's self-evaluation, resulting in 'self-threat'. Similarly, [6] find that the threat of status loss makes individuals conform to norms, reducing their creativity (p. 595). Studies also suggest, albeit inconsistently, that the effect of status loss is uneven across individuals. Some studies suggest that individuals who bore higher status prior to the loss exhibit a more severe drop in performance [7], while others argue that the effect is more pronounced for those who possess intermediate status [6]

Our study contributes to the prior body of work in several ways. First, instead of focusing upon performance changes deriving from status loss, we consider the affected individual's intermediate behavioral response by observing their resource expenditure. For an individual, spending scarce resources is a far more deliberate action than performance, for which there are several "potential impediments outside the control of the individual" [9; p.990]. Thus, from a behavioral standpoint, studying the impact of status loss on resource expenditure is substantially more meaningful, potentially yielding more actionable managerial and policy implications. Second, we relax an important boundary condition pertaining to the effect of status loss. A common consideration in most prior work on status loss is that "work demands do not stop after losing status" [7; p. 255]. However, there are numerous settings in which 
this is not the case, with voluntary, unpaid work being the most obvious example. Hence, our focus upon online software coding contests, wherein participation is entirely voluntary. In this setting, individuals retain a near-complete autonomy in deciding their course of action after a status loss. Our study thus provides an empirical test of theoretical predictions of status loss in a qualitatively different context [10], wherein behavioral responses are not constrained by the demands of gainful employment. Lastly, exploiting a natural experiment, in the form of an exogenous shock to an established status hierarchy, we are able to tease out the effects of status loss, isolating them from any endogenous association between naturally occurring status loss and subsequent resource expenditure and performance. Thus, we are able to overcome the persistent issue of endogeneity between the symbolic effect of status and the actual underlying quality [11].

Our study context is CodeForces.com, an online platform that hosts software programming contests. The platform categorizes users into nine mutually exclusive classes, based on metrics of individuals' past contest performance. For instance, the lowest group is referred to as Newbie and the highest as an International Grandmaster. This categorization creates tiers in the participant pool, demarcating status. Typically, in online contest platforms, individuals who are placed in higher categories, attract greater prestige, admiration from fellow developers, and even signal expertise to potential recruiters. In October 2015, the platform restructured the classification scheme, exogenously reassigning some individuals to a lower category, independent of any changes in their resource expenditure or performance. We leverage this natural experiment to identify the effects of status loss. Estimating a Difference-In-Differences (DID) specification with the contest and user fixed effects, we find that status loss leads to an approximate $70 \%$ increase in a user's subsequent resource expenditure.

Our findings indicate that arbitrarily status loss induces individuals to attempt to reacquire it. Thus, while prior work shows that performance outcomes may deteriorate after the status loss [6], we find evidence to the contrary; that resource expenditure spikes in response. As noted above, our contradictory results are likely attributable to some combination of our unique identification of the effects, and the voluntary nature of resource expenditure in our study context.

\section{Background Literature}

\subsection{Status and Status Seeking}

Status is defined as "one's relative standing in a social hierarchy as determined by respect, deference and social influence" [12; p. 281]. The concept has attracted considerable research attention across a number of settings, dealing with work in both collective [13] and competitive tasks [2, 14]. Existing work shows that individuals actively seek high status because it provides "access to power and resources, and therefore is pursued consciously in many situations" [15; p. 105]. More importantly, studies consistently show that behavior is driven by statusseeking, although there is some disagreement as to whether attaining high status itself is the end goal [15], or whether individuals strive for high status in order to achieve other outcomes [16]. Some instances of status-seeking behavior include product purchase decisions [17] and participation in online communities $[18,19]$.

\subsection{Performance Implications of Status Loss}

Although individuals seek status through concerted efforts, the hazard of losing status is ever present [4], and individuals are sensitive to this [20]. One can lose status in many ways, ranging from a highly publicized scandal to demotion in a job. Surprisingly, however, "the literature on status processes has largely overlooked the phenomenon of status loss" [21, p. 477]. In the emerging yet limited body of work to date, the focus has typically been on the performance implications of the status loss. [7] argue that after a status loss, an individual may experience self-threat and as a result, a breakdown of the information processing necessary to execute their tasks, leading to poorer performance (p. 226). Similarly, [5] found that status loss among investment brokers, stemming from the creation of new analyst ranking categories in an established status hierarchy, resulted in weaker response and reduced attention from investors, leading to performance declines. [6] argued that the threat of status loss can force individuals to conform to norms, lowering subsequent creative output.

While these early studies provide encouraging results, they also suffer from certain limitations, not the least of which relates to the use of market or labor performance as the outcome of interest. Performance, as conceptualized in the present work on status loss, is not solely a product of a focal individual's behavioral response to losing his or her status; rather, it is a product of that behavioral response, as well as the response of other stakeholders in the market, e.g., consumer perceptions. This argument is related to the 
ongoing debate about the view of performance as a behavior rather than performance as an outcome. While the advocates of the latter view suggest that "performance is the result of what has been done", proponents of the 'performance as a behavior' stance argue that "performance is in the doing" [9; p. 990]. Thus, to understand a focal actor's own response to status loss, it is necessary to consider participationbased measures. To this end, we deviate from prior work by examining the effect of status loss on the individual's resource expenditure, rather than his or her performance outcomes.

\subsection{Behavioral Autonomy after Status Loss}

One of the key assumptions in the prior literature is that "work demands don't stop after status loss" [7; p. 224]. For example, [21] examine the effect of status loss among employees of a French multinational. Clearly, the employees are required to perform their duties regardless of their status. The same can be said about studies involving members of the British Parliament [4], and financial investment analysts [5]. We argue that the presence of persistent work demands limits the behavioral autonomy of individuals, and thereby constraints their behavioral response to status loss. That is, an employee is constrained from simply ceasing their professional duties following a status loss, out of a need to maintain gainful employment. The degree to which that constraint applies will depend on the degree to which a given individual is locked into or dependent upon their employment. By adopting an online contest platform as our empirical setting, we relax this constraint. On these types of platforms, resource expenditure is generally voluntary. A user can simply withdraw from the platform and stop participating if they wish, with minimal cost. As such, the greater behavioral autonomy that online contest platforms afford leaves the door open to extreme behavioral responses, ranging from complete withdrawal to extremely high resource expenditure. As a result, examining the behavioral effects of status loss in such settings is potentially quite informative.

\subsection{Estimating Causal Effects of Status Loss}

Finally, we are also conscious of the endogeneity issues that plague the status loss literature. Status is often predicated on past performance and resource expenditure. Thus, the status loss may very well reflect on-going changes in a person's behavior, rather than cause those changes. This reverse causality creates obvious problems in identifying the effect of status changes [11]. As some recent empirical evidence on the performance outcomes of status changes suggests, accounting for this endogeneity can yield drastically different effect estimates [22]. In the present work, we overcome this challenge while examining the resource expenditure response to status loss, by leveraging an exogenous shock to the status hierarchy on the platform we study. The shock was such that individuals lost status suddenly and in a manner independent of their prior performance or resource expenditure.

\section{Methodology}

\subsection{Empirical Context}

We study the impact of individuals' loss of status on subsequent resource expenditure, in an online contest setting: http://codeforces.com/. The platform hosts regular time-bound competitions in which participants can submit multiple solutions, with the objective of improving their contest score against a pre-defined software scoring algorithm. Based on past contest performance, the platform rates participants in a manner similar to the wellestablished Elo ratings used in chess and other competitive sports

(https://en.wikipedia.org/wiki/Elo_rating_system).

Conceptually, the notions of status and contest are compatible because "central to both concepts are hierarchical orders and their impact on human behavior" [23, p.120]. Moreover, there is growing interest in studying status in a variety of offline competitive settings $[14,23]$. Online contest platforms have become quite common in recent years [24] and hence provide a novel opportunity to study status in a digital competitive space of great practical relevance.

From a behavioral viewpoint, each participant on the contest platform faces a two-staged, sequential decision: whether to submit to a given contest and how many submissions to make. Typically, a participant makes multiple submissions. In the present study, we model these outcomes.

\subsection{Status Hierarchy on CodeForces.com}

The platform is highly transparent in its assignment of participants to distinct status categories. First, based on the participant's current rating, she is assigned to one of two divisions ${ }^{1}$. A participant can submit solutions in contests hosted

\footnotetext{
${ }^{1}$ As of May 2018, subsequent to our data collection period, a third division has been introduced.
} 
either in their own division or in contests that are made open to both divisions. Second, depending on the participant's rating, he or she is assigned to a color-coded group, such that across the platform, the participant's username is consistently displayed in the associated color. Given that a participant is assigned to a status category based on her past performance, others readily associate her expertise and skill levels with the color in which her username appears. Given that the color scheme has been in existence since November 2011, there has been ample opportunity for these colors to become salient markers of the participant's status on CodeForces.com. Moreover, anecdotal evidence in the form of discussion thread postings in the Codeforces.com user forum suggests that users do in fact ascribe status to these color coding. As such, our conceptualization of status as conveyed through distinct, prominent categories is consistent with prior work on status loss [5].

We collected information on all contests, submissions, participants and their time-varying characteristics, including ratings and color status, through February 2010 to January 2018 using the platform's Web application programming interface.

\subsection{Model Specification}

To empirically examine the role of status changes on resource expenditure, we began by analyzing the association between naturally occurring changes in participants' color status, i.e., changes deriving from historical performance, and participants' subsequent participation and resource expenditure. Of course, this relationship is likely subject to endogeneity, as described earlier. Most obviously, an individual's relative performance in a contest at time $t$ may decline, resulting in a status loss, because of fading interest or engagement in the platform. That fading interest also drives subsequent further declines in resource expenditure and performance, and eventual exit from the platform altogether. Intuitively, this data-generating process would yield a negatively biased estimate of the relationship between status loss and subsequent participation and submission volumes. As such, this initial, unidentified analysis is performed primarily to serve as a point of comparison, illustrating the importance of separately identifying the effect of changes in status from that of changes in performance.

We construct our panel data set based on a sliding window of inclusion, such that a user-contest observation was included only if an individual submitted to at least one contest in the two weeks preceding or following the observation. This was done to ensure the user was actively deciding to submit or not.

We estimated multiple regression specifications. First, we considered the binary participation decision, and thus estimated a Logistic regression and Linear Probability Model (LPM), specifying the binary indicator of submission to a given contest, $t$, by a given individual, $i$, Submission $(i, t)$, as a function of recent status changes. For any given contest-user pair, we respectively define StatusLoss $(i, t)$ and $\operatorname{StatusGain}_{(i, t)}$ in that observation as an indicator of whether the user lost or gained status as a result of his or her most recent prior contest participation

Individual fixed effects and contest fixed effects were included to account for the potential confounding effects of time-invariant attributes associated with users and contests. Equation (1) reflects the LPM specification we estimate, which is also analogous to the logistic regression model. Here, $i$ indexes users and $t$ indexes contests. User fixed effects are represented by $\delta_{i}$, and contest fixed effects by $\tau_{t}$. Finally, $\varepsilon_{(i, t)}$ is our error term.

$$
\begin{aligned}
\operatorname{Submission}_{(i, t)=} & \delta_{i}+\tau_{t}+\operatorname{StatusLoss}_{(i, t)}+ \\
& \operatorname{StatusGain}_{(i, t)}+\varepsilon_{(i, t)} \\
& \text { Equation (1) }
\end{aligned}
$$

Subsequently, we replaced the dependent variable with the count of submissions by individual $i$ to contest $t$. We then repeated the analysis using Poisson regression incorporating individual and contest fixed effects.

\subsection{Recovering the Causal Effect of Status Loss}

As articulated earlier, status loss stemming from the individual's performance outcomes in a prior contest is a function of resource expenditure, and thus likely to be endogenous to individual's subsequent participation and resource expenditure in later contests. This problem has been a persistent empirical challenge in the status literature [11]. To address this issue and to obtain the causal effect of status loss on subsequent resource expenditure, we exploit a natural experiment that resulted from the platform's decision to suddenly alter the existing status categories. On the $1^{\text {st }}$ of October, 2015, the platform modified its color grouping system overnight. Although there were some indications of an impending change, the specific details and timing were never revealed beforehand. Specifically, the color status indicators were changed to incorporate a new color group, leading to a change in the composition of all groups situated above it in the 
hierarchy. Figure 3.3a provides the preexisting set of categories while Figure $3.3 \mathrm{~b}$ depicts the status categories post the exogenous change. Compared to Figure 1, one can notice the change in the Rating Range for each category along with 2 new categories added in Figure 2

\begin{tabular}{|l|l|l|l|}
\hline Ratings Range & Color & Title & Division \\
\hline $2600+$ & Red & International grandmaster & 1 \\
\hline $2200-2599$ & red & Grandmaster & 1 \\
\hline $2050-2199$ & Orange & International master & 1 \\
\hline $1900-2049$ & Orange & Master & 1 \\
\hline $1700-1899$ & Violet & Candidate master & 1 \\
\hline $1500-1699$ & Blue & Expert & 2 \\
\hline $1350-1499$ & Green & Specialist & 2 \\
\hline $1200-1349$ & Green & Pupil & 2 \\
\hline $0-1199$ & Gray & Newbie & 2 \\
\hline
\end{tabular}

Figure 1: Color categories before the shock (http://codeforces.com/blog/entry/3064

\begin{tabular}{|c|c|c|c|c|c|}
\hline Rating Bounds & Color & Title & Division & Number & Number (by color) \\
\hline $2900+$ & Red & Legendary Grandmaster & 1 & 4 & \multirow[t]{3}{*}{183} \\
\hline $2600-2899$ & Red & International Grandmaster & 1 & 46 & \\
\hline $2400-2599$ & Red & Grandmaster & 1 & 133 & \\
\hline $2300-2399$ & Orange & International Master & 1 & 163 & \multirow[t]{2}{*}{380} \\
\hline $2200-2299$ & Orange & Master & 1 & 217 & \\
\hline $1900-2199$ & Violet & Candidate Master & 1 & 1253 & 1253 \\
\hline $1600-1899$ & Blue & Expert & 2 & 5095 & 5095 \\
\hline $1400-1599$ & Cyan & Specialist & 2 & 8202 & 8202 \\
\hline $1200-1399$ & Green & Pupil & 2 & 5736 & 5736 \\
\hline $0-1199$ & Gray & Newbie & 2 & 2319 & 2319 \\
\hline
\end{tabular}

Figure 2: Color categories after the shock (http://codeforces.com/blog/entry/20638)

Of the 14,060 active users at the time of the natural experiment, approximately 7,900 lost their status (moved to a lower-ranked color group), and another approximately 2,000 were assigned to a completely new, ostensibly higher-status color (Cyan). The following participant postings/comments, taken from the platform discussion forum shortly after the change was implemented, suggest that individuals who experienced a downward shift in their category experienced a sense of loss (http://codeforces.com/blog/entry/20638)

"Well, I'm feeling kinda empty :-(, but alright, have to be purple again"
"I became yellow [Orange] in CF because of the revolt of colors. Sad! :("

"Participated in yesterday's contest div2 and solved problem A-today I am Cyan. Lol. dafaq!"

We focus on the 7,874 participants who saw their status reduced. Although the subjects who received a new color could be argued to have experienced an exogenous increase in their status, this interpretation is questionable, because status theory argues that status is a product of norms and perceptions constructed and reinforced over time [25]. Given the overnight introduction of this new status tier, it appears unlikely that it would deliver a clear increase in status to affected participants.

Given that the change to the color-coding scheme was sudden, and its adjustments were in no way implemented as a function of participant performance or rating dynamics, it constitutes a clean natural experiment, which we leverage to evaluate the causal impact of status loss on subsequent participation and resource expenditure. Equation (2), which reflects our natural experiment estimation, is similar to Equation (1), except that the subscripts have been modified to reflect a focus on a pair of contests for each participant, $i$, namely the contest immediately preceding the change, and that immediately following the change. Moreover, the StatusLossExo $(i, t)$ and StatusGainExo $(i, t)$ variables, in this case, reflect indicators of whether participant $i$ has experienced an exogenous shift in his or her color tier, in the present period, as a result of the platform change (this amounts to the interaction term in our $\mathrm{DiD}$ specification). Additionally, we replace the vector of contest fixed effects with a simple post dummy. Value of the dummy variable, Post is 1 in the all the observations of the contest that took place immediately after the shock. As a result, post dummy correlates with contest features.

$$
\begin{aligned}
\text { Submission }_{(i, t)=}= & \delta_{i}+\text { Post }_{t}+\text { StatusLossExo }_{(i, t)}+ \\
& \text { StatusGainExo }_{(i, t)}+\varepsilon_{(i, t)}
\end{aligned}
$$

Equation (2)

The primary coefficient of interest in this regression is that associated with StatusLossExo, the difference in differences estimate associated with status loss. We include all users, noting that inactive users will be unresponsive to the exogenous shock, as they will not be aware of it. Accordingly, including these individuals in our regression analyses will merely make it more difficult for us to detect statistically significant effects from the treatment. As such, any resulting effects we identify can reasonably 
be viewed as conservative estimates. Once again, after estimating the binary response models, we repeat the process employing Poisson regression on the count of submissions.

\subsection{Data}

Data used in our (endogenous) panel regression sample is comprised of all individual-contest observations that preceded our natural experiment. The estimation sample pertaining to our natural experiment, in contrast, includes exactly two observations per individual; one for the last contest the individual could conceivably have submitted to prior to the shock, and a second for the next contest conducted immediately following the shock. We adopt this approach because the effect of status loss can be teased out most cleanly by observing the immediate contests. If the time window around the shock is expanded, the effects are likely to be confounded by other unobserved determinants. Moreover, we don't observe any acute deviations between the contests that occurred immediately before and after the shock, and the rest of the contests represented in the dataset. For instance, the contests used in the DiD estimation have an average duration of 7650 seconds while the average duration of all the contests is 7579 seconds.

Descriptive statistics for our panel regression sample are presented in Table 1, and those for our natural experiment sample are presented in Table 2. Considering the panel data set, in Table 1, we see that the average individual-contest pair includes 2.89 submissions, but that the distribution is highly skewed, with a maximum value of 211 submissions to a single contest.

Moreover, even with our sliding window of inclusion, based on individuals submitting to at least one contest in the two weeks preceding or following a contest, we still see that roughly $50 \%$ of the individual-contest observations involve zero submissions. This observation helps justify our consideration of both the binary submission decision (participation), separate from the count of submissions (effort). The status change dummies indicate that both events are quite frequent, with status loss preceding 29\% of individual-contest observations, and status gain preceding $17 \%$. Considering the Natural Experimental sample, in Table 2, we see similar patterns.

Table 1: Descriptive statistics for contestantcontest panel

\begin{tabular}{|l|l|l|l|l|}
\hline $\mathrm{N}$ & Mean & Min & Max & SD \\
\hline
\end{tabular}

\begin{tabular}{|l|l|l|l|l|l|}
\hline $\begin{array}{l}\text { Submit } \\
\text { Count }\end{array}$ & 621,361 & 2.89 & 0 & 211 & 4.75 \\
\hline StatusLoss & 594,878 & 0.29 & 0 & 1 & 0.45 \\
\hline StatusGain & 594,878 & 0.17 & 0 & 1 & 0.38 \\
\hline
\end{tabular}

Table 2: Descriptive statistics for natural experiment

\begin{tabular}{|l|l|l|l|l|l|}
\hline & $\mathrm{N}$ & Mean & Min & Max & SD \\
\hline $\begin{array}{l}\text { Submit } \\
\text { Count }\end{array}$ & 28,120 & 0.89 & 0 & 47 & 2.65 \\
\hline $\begin{array}{l}\text { StatusLoss } \\
\text { Exo }\end{array}$ & 28,120 & 0.28 & 0 & 1 & 0.45 \\
\hline $\begin{array}{l}\text { StatusGain } \\
\text { Exo }\end{array}$ & 28,120 & 0.07 & 0 & 1 & 0.26 \\
\hline
\end{tabular}

\section{Results}

The results of our binary regression models are presented in Tables 3 (LPM) and 4 (Logit). Looking first to the panel regression results in Table 3, we find that loss of status as a result of the last active contest participation is associated with a $1.3 \%$ increase in the probability that an individual submits to a subsequent contest. Considering our count model, in the $1^{\text {st }}$ column of Table 5, we see a similar result, indicating an approximate $2.3 \%$ increase in the volume of submissions to a subsequent contest, following a status loss.

Recall, however, that these estimations are likely to be downward biased because a typical status loss is quite likely to be reflective of a pre-existing downward trend in an individual's engagement with or interest in the platform. Accordingly, we might expect that the effects are more positive. Indeed, this is exactly what we observe when we shift focus to the results of our natural experiment estimations. The observed shift in the effects status loss underscores the role of panel data results as a basis of comparison.

Returning to Table 4, we observe larger coefficients in each case. Focusing on the logistic regression result, which is arguably the more reliable of the two, e.g., given that LPMs do not constrain the model to yield predictions in the 0-1 range, we see an order of magnitude increase in the estimated coefficient on the loss of status. Similarly, in the second column of Table 5, we see an estimated positive effect of 0.532 , showing a $20 \mathrm{x}$ increase in the estimated coefficient, which translates to a nearly $70 \%$ increase in the rate of contest submission.

Table 3: Status loss effect on the decision to submit (linear probability model) 


\begin{tabular}{|l|l|l|}
\hline & $\begin{array}{l}\text { Contest-Participant } \\
\text { Panel }\end{array}$ & $\begin{array}{l}\text { Natural } \\
\text { Experiment }\end{array}$ \\
\hline StatusLoss & $0.013^{* * *}(0.001)$ & $0.016^{+}(0.008)$ \\
\hline StatusGain & $-0.008^{* * *}(0.002)$ & $0.003(0.011)$ \\
\hline User FE & Yes & Yes \\
\hline Contest FE & Yes & Yes \\
\hline N & 592822 & 28120 \\
\hline Adj. $\mathrm{R}^{2}$ & 0.148 & 0.273 \\
\hline
\end{tabular}

Cluster robust standard errors reported in the parentheses; OLS estimator with two-way fixed effects is used;

${ }^{+} \mathrm{p}<0.10,{ }^{*} \mathrm{p}<0.05,{ }^{* *} \mathrm{p}<0.01,{ }^{* * * *} \mathrm{p}<0.001$

\section{Table 4: Status loss effect on the decision to submit (logit)}

\begin{tabular}{|l|l|l|}
\hline & $\begin{array}{l}\text { Contest- } \\
\text { Participant Panel }\end{array}$ & $\begin{array}{l}\text { Natural } \\
\text { Experiment }\end{array}$ \\
\hline StatusLoss & $0.066^{* * *}(0.008)$ & $0.646^{* * *}(0.098)$ \\
\hline StatusGain & $-0.042^{* * * *}(0.009)$ & $0.096(0.122)$ \\
\hline User FE & Yes & Yes \\
\hline Contest FE & Yes & Yes \\
\hline N & 594878 & 5348 \\
\hline
\end{tabular}

Cluster robust standard errors reported in the parentheses; Logit estimator with two-way fixed effects is used; ${ }^{+} \mathrm{p}<0.10,{ }^{*} \mathrm{p}<0.05,{ }^{* *} \mathrm{p}<0.01,{ }^{* * *} \mathrm{p}<0.001$

\section{Table 5: Status loss effect on submission count}

\begin{tabular}{|l|l|l|}
\hline & $\begin{array}{l}\text { Contest-Participant } \\
\text { Panel }\end{array}$ & $\begin{array}{l}\text { Natural } \\
\text { Experiment }\end{array}$ \\
\hline StatusLoss & $0.023^{* * *}(0.005)$ & $0.532^{* * *}(0.071)$ \\
\hline StatusGain & $-0.028^{* * *}(0.005)$ & $-0.053(0.089)$ \\
\hline User FE & Yes & Yes \\
\hline Contest FE & Yes & Yes \\
\hline $\mathrm{N}$ & 569348 & 6810 \\
\hline
\end{tabular}

Cluster robust standard errors reported in the parentheses; Poisson estimator with two-way fixed effects is used; ${ }^{+} \mathrm{p}<$ $0.10,{ }^{*} \mathrm{p}<0.05,{ }^{* *} \mathrm{p}<0.01,{ }^{* * *} \mathrm{p}<0.001$

\section{Conclusion}

We have presented a novel analysis of status loss effects on individuals' subsequent resource expenditure in the context of an online software coding contest platform, Codeforces.com. Our work departs from the small body of prior work on status loss in three ways. First, we consider a context involving voluntary, unpaid work, where individuals' responses to status loss are not constrained by persistent work demands. That is, individuals have the autonomy to reduce their engagement or exit the market entirely. Second, we focus on the immediate impact upon affected individuals' resource expenditure, rather than changes in performance, noting that performance is a downstream outcome that results from both affected individuals' resource expenditure, as well as perceptions of other stakeholders in the market, e.g., consumers, which may also be influenced by the focal actor's status loss. Third, we attend closely to the issue of causal identification, exploiting a natural experiment in which the platform operator adjusted status markers independent of any changes in individuals' resource expenditure or performance.

We provide evidence that status loss results in large increases in affected individuals' resource expenditure. In short, when individuals experience exogenous status loss, they respond vigorously by attempting to reacquire that status.

Our findings suggest that the mechanisms behind prior findings of performance declines following a status loss are either context-dependent or a result of negative market perceptions dominating any resource expenditure increase on the part of affected workers. These findings have important implications for practice and policy, as they suggest that the optimal approach to managing worker effort in the face of status loss should focus not on inducing effort; rather, they should focus on managing perceptions.

For example, in our setting, market perceptions primarily play a role in career search for affected contestants. Anecdotal evidence suggests that some workers benefit from improved career prospects as a result of their success in these contests. Status shifts that ignore this may do unnecessary harm to contestants if the message to employers is not well managed.

More generally, our findings contribute to the literature on the status loss. In large part, this contribution arises by raising new questions about the nature and mechanisms of status loss effects on worker performance. Our findings suggest that additional work is needed to tease apart resource expenditure from performance outcomes, as a result of the status loss, and to understand the moderating influence of incentive structures, be they job search, financial compensation or intrinsic motivators.

This study has several possible extensions, which may aid in further uncovering the underlying statusbased mechanisms. First, one may assess whether the observed effects of status loss is predicated on the solver's status before the shock. Extant work indeed suggests that those with higher prior status respond to status loss more strongly than those with lower prior status [7] Second, future studies can examine whether the exogenous changes in the status also affects the quality of the output that users generate. Lastly, one 
can also examine the length for which the effect of status loss lasts.

\section{References}

[1] Merton, R. "The Matthew Effect in Science". Science, 159, 1968, pp. 56-63.

[2] Washington, M., \& Zajac, E. "Status Evolution and Competition: Theory and Evidence". Academy of Management Journal, 48(2), 2005, pp. 282-296

[3] Piazza, A., \& Castellucci, F. "Status in Organization and Management Theory." Journal of Management, 40(1), 2014, pp. 287-315.

[4] Graffin, S., Bundy, J., Porac, J., Wade, J., \& Quinn, D. "Falls from Grace and the Hazards of High Status: The 2009 British MP Expense Scandal and Its Impact on Parliamentary Elites". Administrative Science Quarterly, 58(3), 2013, pp. 313-345.

[5] Bowers, A., \& Prato, M., "The Structural Origins of Unearned Status: How Arbitrary Changes in Categories Affect Status Position and Market Impact”. Administrative Science Quarterly. 2017, DOI: https://doi.org/10.1177/0001839217727706

[6] Duguid, M., \& Goncalo, J. "Squeezed in the Middle: The Middle Status Trade Creativity for Focus". Journal of Personality and Social Psychology, 109(4), 2015, pp. 589 603

[7] Marr, J., \& Thau, S. "Falling from Great (and not-sogreat) Heights: How Initial Status Position Influence Performance after Status Loss". Academy of Management Journal, 57(1), 2014, pp. 223-248.

[8] Bendersky, C., \& Shah, N. "The Cost of Status Enhancement: Performance Effects of Individuals' Status Mobility in Task Groups". Organization Science, 23(2), 2012, pp. 308-322.

[9] Beal, D., Cohen, R., Burke, M., \& McLendon, C. "Cohesion and Performance in Groups: A Meta-Analytic Clarification of Construct Relations". Journal of Applied Psychology, 88(6), 2003, pp. 989-1004

[10] Whetten, D., "What Constitutes Theoretical Contribution". Academy of Management Review, 14(4), 1989, pp. 490-495.

[11] Malter, D. "On the Causality and Cause of Returns to Organizational Status: Evidence from the Grands Crus Classés of the Médoc". Administrative Science Quarterly, 59(2), 2014, pp. 271-300.

[12] Ridgeway, c. \& Walker, H. "Status Structures." Sociological Perspectives on Social Psychology, 1995, pp. 281-310. NJ: Pearson Education
[13] Berger, J., Rosenholtz, S., \& Zelditch Jr., M. "Status Organizing Processes". Annual Review of Sociology, 6, 1980, pp. 479-508

[14] Flynn, F., \& Amanatullah, E. "Psyched Up or Psyched Out? The Influence of Co-Actor Status on Individual Performance". Organization Science, 23(2), 2012, pp. 402415.

[15] Huberman, B., Loch, C., \& Önçüler, A. "Status as a Valued Resource". Social Psychology Quarterly, 67(1), 2004, pp.103-114.

[16] Thye, S. "A Status Value Theory of Power in Exchange Relations". American Sociological Review, 65, 2000, pp. 407-432.

[17] Millan, E., \& Mittal, B. "Consumer Preference for Status Symbolism of Clothing: The Case of the Czech Republic". Psychology \& Marketing, 34(3), 2017, pp. 309322.

[18] Roberts, J., Hann, I., \& Slaughter, S. "Understanding the Motivations, Participation, and Performance of Open Source Software Developers: A Longitudinal Study of the Apache Projects". Management Science, 52(7), 2006, 984999.

[19] Lampel, J., \& Bhalla, A. "The Role of Status Seeking in Online Communities: Giving the Gift of Experience". Journal of Computer-Mediated Communication, 12, 2007, $434-455$

[20] Pettit, N, Kevyn Y, Sandra S. "Holding your Place: Reactions to the Prospect of Status Gains and Losses". Journal of Experimental Social Psychology. 46(2), 2010, pp. 396-401

[21] Neeley, T. "Language Matters: Status Loss and Achieved Status Distinction in Global Organizations" Organization Science, 24(2), 2013, pp. 476-497.

[22] Azoulay, P., Stuart, T., \& Wang, Y. "Matthew: Effect or Fable?" Management Science, 60(1), 2013, pp. 92 - 109.

[23] Nippa, M. "On the Need to extend Tournament Theory through Insights from Status Research". In J. L. Pearce (Ed.), Status in Management and Organizations: 2011, pp. 118-152. Cambridge, UK: Cambridge University Press.

[24] Boudreau, K., Lacetera, N., \& Lakhani, K. "Incentives and Problem Uncertainty in Innovation Contests: An Empirical Analysis". Management Science, 57(5), 2011, pp. 843-863.

[25] Ridgeway C. "Status Construction Theory". In Contemporary Social Psychological Theory, ed. P.J. Burke, 2006, pp. 301-23. Stanford: Stanford University Press 